\title{
Fabry Perot Semiconductor Lasers with Periodic Diffused Quantum Wells Structure
}

\author{
C.W. Lo, S.F. Yu and E. H. Li \\ Department of Electrical and Electronic Engineering, \\ The University of Hong Kong, Pokfulam Road, Hong Kong
}

\begin{abstract}
Fabry Perot semiconductor laser with periodic change in the extent of interdiffusion along the longitudinal direction of the Quantum Well active region is proposed to improve the discrimination between cavity modes.
\end{abstract}

The conventional longitudinal-mode discrimination in Fabry Perot(FP) lasers is only provided by the material gain spectrum and the resulting mode discrimination is poor, especially at low output power. In this paper, a FP laser with periodic diffusion Quantum Well (DFQW) structure which serves as the active region is proposed for the first time to improve the longitudinal-mode discrimination.

Figure 1 shows the schematic of a FP laser with periodic DFQW structure. The active region consists of three as-grown and interdiffusion modified $\mathrm{Al}_{0.3} \mathrm{Ga}_{0.7} \mathrm{As} / \mathrm{GaAs}$ single $\mathrm{QW}$ with well width and barrier thickness of $100 \AA$ and $250 \AA$, respectively. The extent of interdiffusion is characterized by a diffusion length, $L_{d} \cdot{ }^{1}$ It is assumed that $L_{d}$ is varied along the longitudinal direction of the QW active region periodically with magnitude equal to 0 and $100 \AA$, respectively. The longitudinal length of each diffused section is $85(\lambda / 4)$ except in the centre of the laser which is $85(\lambda / 2)$ long, where $\lambda$ is the operation wavelength. The total number of diffused sections is equal to 57 and the effective gain peak wavelength of this periodic DFQW active region is around $0.85 \mu \mathrm{m}$.

The gain of the DFQW, $G$, can be expressed as $G(\lambda)=g(\lambda) \ln \left(N / N_{o}(\lambda)\right),{ }^{2}$ where $N$ is the carrier concentration. The gain coefficient, $g$, and the carrier at transparency, $N_{0}$, for $L_{d}=0$ and $100 \AA$ (at $\lambda=0.85 \mu \mathrm{m}$ ) are equal to $662 \mathrm{~cm}^{-1}$ and $2.2822 \times 10^{18} \mathrm{~cm}^{-3}, 137 \mathrm{~cm}^{-1}$ and $101.54 \times 10^{18} \mathrm{~cm}^{-3}$, respectively. The refraction index of the DFQW active region is equal to 3.645 for $\mathrm{L}_{\mathrm{d}}=0 \AA$ and 3.35 for $\mathrm{L}_{\mathrm{d}}=100 \AA^{3}$. The effective refractive index of the corresponding waveguide section with $L_{d}=0$ and $100 \AA$ is equal to 3.4549 and 3.2365 , respectively.

The spontaneous emission power can be calculated by using the power matrix method. ${ }^{4}$ The rate equation of carrier is also employed to relate the injection current, carrier concentration and the optical power in a self-consistent manner.

The amplified spontaneous spectrum of a FP laser with periodic DFQW structure is shown in figure 2. It is shown that as the current increases from $0.97 \mathrm{~J}_{\text {th }}$ to $1.0 \mathrm{~J}_{\text {th }}$, the Bragg band-gap mode reaches threshold and suppresses the other longitudinal modes, where $J_{\text {th }}$ is the threshold current. In addition, the threshold gain margin between main mode and side 
mode of the FP laser with periodic DFQW structure is calculated and is shown to be larger than that of the conventional FP laser by $26 \mathrm{~cm}^{-1}$.

In fact, the periodic DFQW structure is similar to a complex-coupled distributed feedback laser except the dimension of the grating. The length of the grating period of the periodic DFQW structure is about 40 times longer than that of the distributed feedback laser. Figure 3 shows the amplified spontaneous spectrum of the modified FP laser with a wider spectral range. It is noted that only the Bragg band-gap mode near the gain peak dominates the emission spectrum. This is because the effective gain spectral linewidth is narrow enough to discriminate the other longitudinal modes away from the peak gain wavelength. Therefore, single longitudinal mode operation of FP lasers can be achieved with the presence of periodic DFQW structure.

\section{References}

[1] E.H. Li, B.L. Weiss and K.S. Chan, Phys. Rev. B, 46, 15181 (1992).

[2] E.H. Li and K.S. Chan, Electronics Lett., Vol. 29, 1233 (1993).

[3] E.H. Li, B.L. Weiss, K.S. Chan and J. Micallef, Appl. Phys. Lett. 62, 550 (1992).

[4] L.M. Zhang and J.E. Carroll, IEEE J. Quantum Elect., QE-28, 604 (1992)

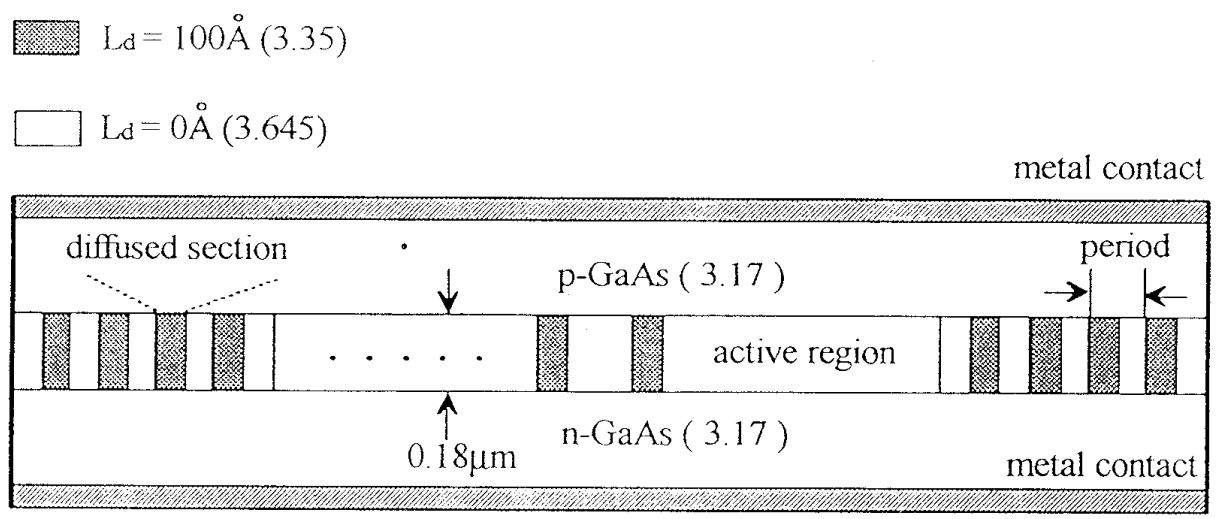

Figure 1. Schematic diagram of the FP laser with periodic DFQW structure along the longitudinal direction of the active region. The power reflection coefficients at the facets are equal to 0.30 . 


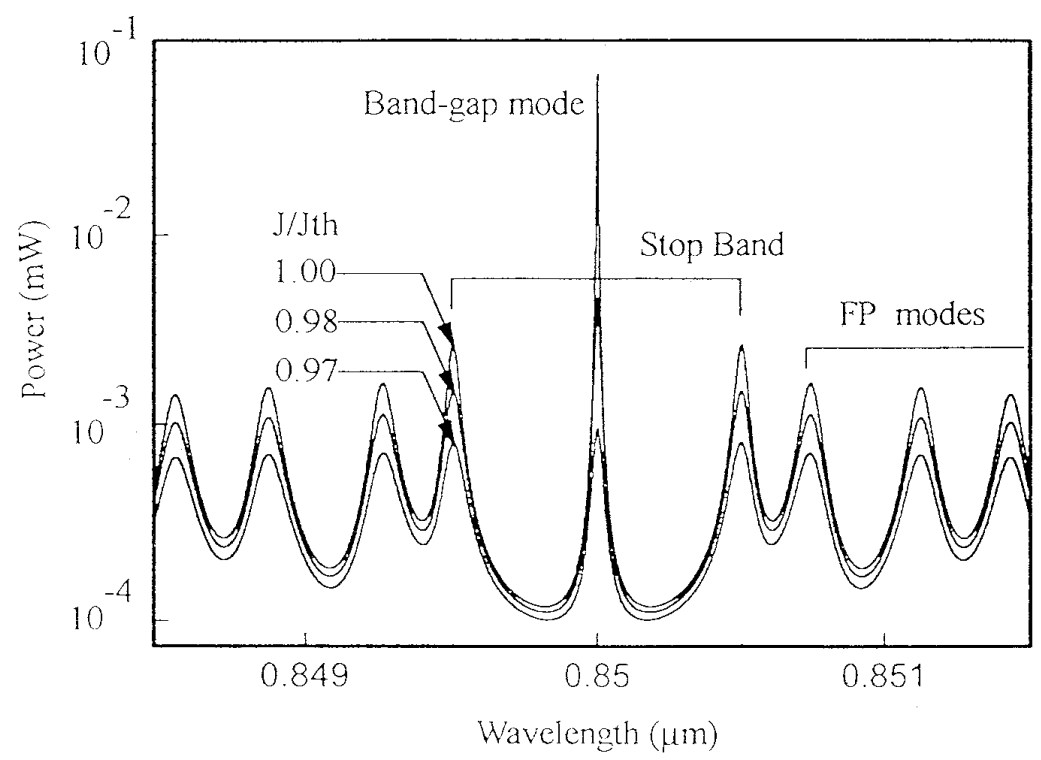

Figure 2. Amplified spontaneous spectrum of the FP laser with periodic DFQW structure The laser is biased at $0.97 \mathrm{Jth}, 0.98 \mathrm{Jth}$ and $1.0 \mathrm{Jth}$.

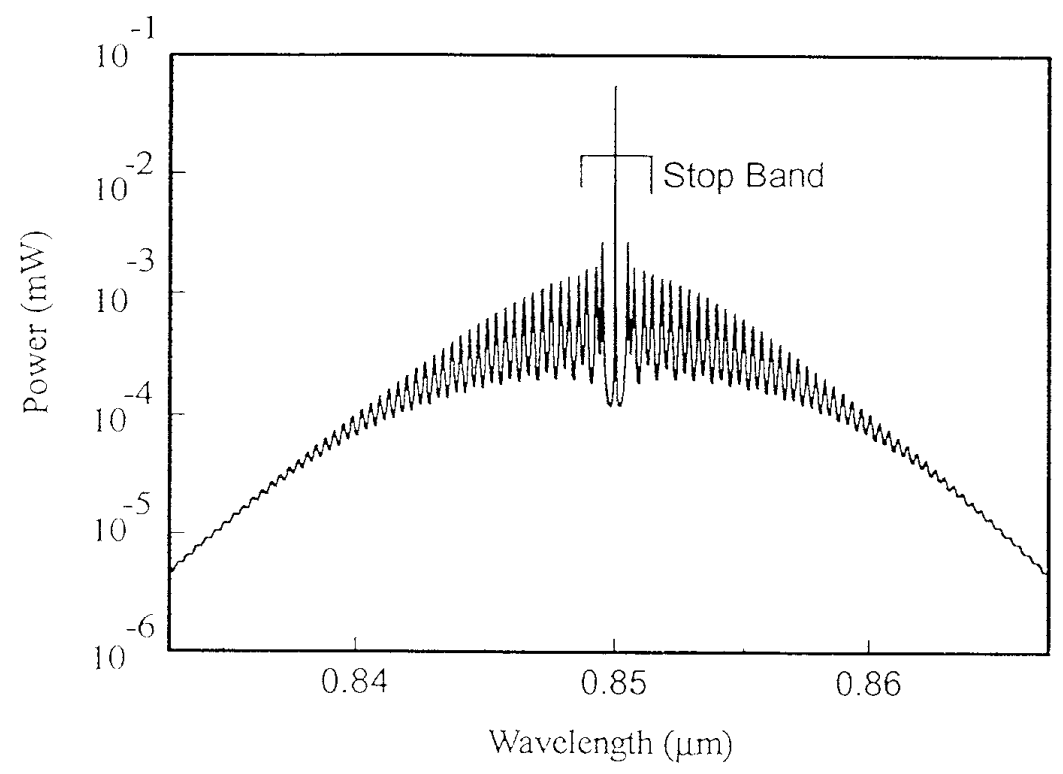

Figure 3. Amplified spontaneous spectrum of the FP laser with periodic DFQW structure. The laser is biased at the threshold. 